\title{
Using routinely recorded data in a UK RCT: a comparison to standard prospective data collection methods
}

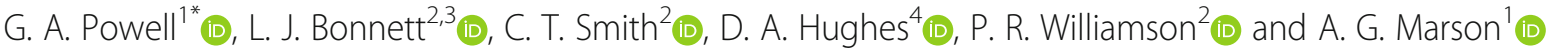

\begin{abstract}
Background: Routinely recorded data held in electronic health records can be used to inform the conduct of randomised controlled trials (RCTs). However, limitations with access and accuracy have been identified. Objective: Using epilepsy as an exemplar condition, we assessed the attributes and agreement of routinely recorded data compared to data collected using case report forms in a UK RCT assessing antiepileptic drug treatments for individuals newly diagnosed with epilepsy.

Methods: The case study RCT is the Standard and New Antiepileptic Drugs II (SANAD II) trial, a pragmatic, UK multicentre RCT assessing the clinical and cost-effectiveness of antiepileptic drugs as treatments for epilepsy. Ninety-eight of 470 eligible participants provided consent for access to routinely recorded secondary care data that were retrieved from NHS Digital Hospital Episode Statistics $(N=71)$ and primary and secondary care data from The Secure Anonymised Information Linkage Databank ( $N=27)$. We assessed data items relevant to the identification of individuals eligible for inclusion in SANAD II, baseline and follow-up visits. The attributes of routinely recorded data were assessed including the degree of missing data. The agreement between routinely recorded data and data collected on case report forms in SANAD II was assessed using calculation of Cohen's kappa for categorical data and construction of Bland-Altman plots for continuous data.
\end{abstract}

Results: There was a significant degree of missing data in the routine record for 15 of the 20 variables assessed, including all clinical variables. Agreement was poor for the majority of comparisons, including the assessments of seizure occurrence and adverse events. For example, only 23/62 (37\%) participants had a date of first-ever seizure identified in routine datasets. Agreement was satisfactory for the date of prescription of antiepileptic drugs and episodes of healthcare resource use.

Conclusions: There are currently significant limitations preventing the use of routinely recorded data for participant identification and assessment of clinical outcomes in epilepsy, and potentially other chronic conditions. Further research is urgently required to assess the attributes, agreement, additional benefits, cost-effectiveness and 'optimal mix' of routinely recorded data compared to data collected using standard methods such as case report forms at clinic visits for people with epilepsy.

(Continued on next page)

\footnotetext{
* Correspondence: gpowell@liv.ac.uk

'Department of Molecular and Clinical Pharmacology, Clinical Sciences

Centre, Lower Lane, Fazakerley, Liverpool L9 7LJ, UK

Full list of author information is available at the end of the article
}

C C The Author(s). 2021 Open Access This article is licensed under a Creative Commons Attribution 4.0 International License, which permits use, sharing, adaptation, distribution and reproduction in any medium or format, as long as you give appropriate credit to the original author(s) and the source, provide a link to the Creative Commons licence, and indicate if changes were made. The images or other third party material in this article are included in the article's Creative Commons licence, unless indicated otherwise in a credit line to the material. If material is not included in the article's Creative Commons licence and your intended use is not permitted by statutory regulation or exceeds the permitted use, you will need to obtain permission directly from the copyright holder. To view a copy of this licence, visit http://creativecommons.org/licenses/by/4.0/ The Creative Commons Public Domain Dedication waiver (http://creativecommons.org/publicdomain/zero/1.0/) applies to the data made available in this article, unless otherwise stated in a credit line to the data. 
(Continued from previous page)

Trial registration: Standard and New Antiepileptic Drugs II (SANAD II (EudraCT No: 2012-001884-64, registered 05/ 09/2012; ISRCTN Number: ISRCTN30294119, registered 03/07/2012))

Keywords: Routine data, Administrative data, Agreement, Randomised controlled trial

\section{Background}

There is great expectation that the analysis of routinely recorded healthcare data will provide rapid and efficient answers to healthcare questions and be a vehicle to generate health and wealth for the UK, exemplified by the recent UKRI investment in Health Data Research UK [1] and collaboration between Novartis and National Health Service England to conduct a novel large scale clinical trial using healthcare system data [2]. It is vital therefore that we understand their utility in clinical trials in common chronic diseases, epilepsy being the exemplar in this paper.

Routinely recorded data can be defined as data that are routinely recorded for specific, defined primary purposes, other than audit or research [3]. Data regarding clinical care are routinely documented in electronic medical records and stored in administrative healthcare databases in the UK $[4,5]$.

Routinely recorded data have established use in retrospective observational studies such as record linkage population studies, but their use in randomised controlled trials (RCTs) is less well established. RCTs remain the gold standard for assessing the efficacy and effectiveness of treatments in healthcare [6] and publicly funded, pragmatic RCTs typically provide longer-term outcome data to inform chronic disease management. However, the majority of RCTs are time-consuming and resource-intensive as clinicians typically assess participants at clinic visits and record trial data on case report forms. If a trial is assessing outcomes that are important to participants, such as a core outcome set $[7,8]$, one might expect relevant data to be recorded routinely.

Routinely recorded data have been used to inform judgements about the feasibility, sample size and recruitment targets in RCTs [9], measure certain participant outcomes [10-12] such as mortality and inform health economic analyses [13]. Routinely recorded data are a potential source of data for a wider range of clinical outcomes, and their use could greatly improve the efficiency of clinical research $[5,10,14]$, reducing the burden to participants and researchers [15]. Furthermore, data from non-clinical routine sources may inform outcomes beyond the standard RCT assessments of clinical efficacy and effectiveness. For example, cost data (such as the use of healthcare resources) and socio-economic data (such as employment and means-tested benefits data) may inform health economic analyses and the assessment of the broader societal impact of healthcare interventions.
Limitations in feasibility, accessibility and accuracy have been identified [16, 17]. For example, the accuracy of routinely recorded data in identifying incident cases may be reduced compared to prevalent cases which may impact on the utility of routinely recorded data to identify individuals with 'new' diagnoses, a frequent target group for RCTs. Furthermore, routinely recorded data may have limitations in identifying recurrent events compared to single events. For example, it may be expected that the identification of stroke would be of greater accuracy than the identification of seizure occurrences.

The accuracy of diagnosis of epilepsy using routinely recorded healthcare data compared to an independent review of patients' medical records has been assessed [18]. However, there is scant evidence of the assessment of accuracy or agreement compared to standard methods of data collection employed in prospective research, such as the record of data on Case Report Forms (CRFs).

Routinely recorded data are being used increasingly in prospective research, including RCTs, without evidence of an appraisal for this purpose [19]. An assessment of the attributes and agreement of routinely recorded data compared to data collected using standard prospective methods is therefore urgently required.

\section{Objective}

To assess the attributes and agreement between routinely recorded data and data collected using case report forms in a UK pragmatic RCT assessing antiepileptic drug treatments for individuals newly diagnosed with epilepsy.

\section{Methods}

The case study RCT is the Standard and New Antiepileptic Drugs II (SANAD II (EudraCT No: 2012-001884-64, ISRCTN Number: 30294119)) trial, a pragmatic, UK multicentre RCT assessing the clinical and cost-effectiveness of selected antiepileptic drugs as first-line treatments for newly diagnosed epilepsy.

Individuals with newly diagnosed epilepsy participating in SANAD II, aged 16 years or older and with a minimum of 12 months follow-up, were eligible for inclusion in this study. Participants were sent a study invitation via post and asked to sign a consent form. One further postal invitation was sent if there was no initial response. 
Routinely recorded data were retrieved from NHS Digital [20], which included data for episodes of patient contact with NHS secondary care in England and from The Secure Anonymised Information Linkage Databank (SAIL) [21], including access to data for episodes of patient contact with NHS secondary care and in selected cases primary care for patients in Wales. All datasets used clinical coding systems, the inpatient and outpatient datasets using the International Statistical Classification of Diseases and Related Health Problems (ICD) 10 [22], the primary care dataset using UK READ Codes [23] and the emergency datasets using unique coding systems. The study was reviewed and approved by the North of Scotland Research Ethics Service and Health Research Authority (29/01/16, REC reference: 16/NS/ 0007, Protocol number: UOL001183, IRAS project ID: 189002).

To permit assessment of the attributes of routinely recorded data and agreement compared to data collected using case report forms, data variables relevant to each of the following aspects were identified or constructed from the available datasets:

- The identification of individuals meeting the inclusion criteria and eligible for recruitment

- Variables included 'Age', 'Date of First-Ever Seizure' and 'Date of Diagnosis of Epilepsy'

- The collection of data relevant to the baseline RCT assessment

- Variables included 'Classification of Seizures' and 'Clinical Investigation Results'

- The collection of data relevant to the follow-up RCT assessments

- Variables included 'Date of First Follow-Up Seizure', 'Adverse Events' and 'Planned and Unplanned Healthcare Attendances' together with the constructed outcomes 'Time to First Follow-Up Seizure' and 'Time to 12-Month Remission'

An algorithmic approach was developed for each variable, using knowledge of the coding systems, clinical behaviours and organisational pathways. Similar approaches utilising the clinical interpretation of routinely recorded data have previously been used in studies assessing seizures [24] and in other disease areas in the UK [25-27]. Diagnostic codes indicating the occurrence of relevant events were specified a priori and the 'best-case' dataset was used in the analysis, constructed using the available data from all relevant primary and secondary care routine datasets. Throughout the analyses, the included participants were analysed as a complete cohort, without reference to antiepileptic drug (AED) prescribed or SANAD II study treatment arm. The algorithms developed for each variable together with the diagnostic codes are presented as Supplementary Figures 1-4 and Supplementary Tables 1-6. Figure 1 presents as an example the algorithm for the identification of seizure occurrence.

Participants study data recorded using standard methods in SANAD II were retrieved. Data were captured on CRFs at baseline and regular follow-up intervals (3, 6, 9 and 12 months thereafter).

Assessment of the attributes of routinely recorded data included identifying the degree of missing data compared to data collected using case report forms.

A statistical assessment of the agreement was completed. Bland-Altman methods [28] were used to assess the agreement between paired continuous data and acceptable clinical limits of agreement were specified a priori, informed by clinical discussion. The Difference and Mean between datasets were computed. Bland Altman Plots were constructed with the Difference variable plotted on the $Y$ axis and the Mean variable plotted on the $X$ axis. The mean of the Difference variable was plotted and the 95\% confidence limits of agreement were calculated and discussed in the context of the specified acceptable clinical limits of agreement [28]. Time to event outcomes were assessed using Kaplan-Meier curves and a log-rank test was performed with $P$ value < 0.05 indicating a statistically significant difference. To assess agreement between paired, nominal categorical datasets and cross tabulations were presented followed by calculation of Cohen's kappa and a $P<0.05$ indicated the level of agreement defined by kappa being significant [29]. All analyses were performed in SPSS, version 22.

The STROBE Checklist is the most relevant, available research reporting checklist and has been referred to when drafting this manuscript, the summary flowchart included as an additional file.

\section{Results}

Four hundred and seventy participants in SANAD II were aged 16 years or older with a minimum of 12 months follow-up, fulfilling the inclusion criteria (April 2016). Ninety-eight participants provided consent to participate in this study, 55 males and 43 females with a mean age of 50. Demographics were similar for the 372 patients not providing consent. Routinely recorded data were requested for 71 participants resident in England, with data available in inpatient, outpatient, emergency and critical care datasets and 27 participants resident in Wales, with data available in inpatient, outpatient and emergency datasets. Primary care data were available for a subset of 23 participants resident in Wales.

Table 1 summarises the attributes of the available data in primary and secondary care sources for selected variables in the 23 participants in whom data from both sources were available. As demonstrated, secondary care 


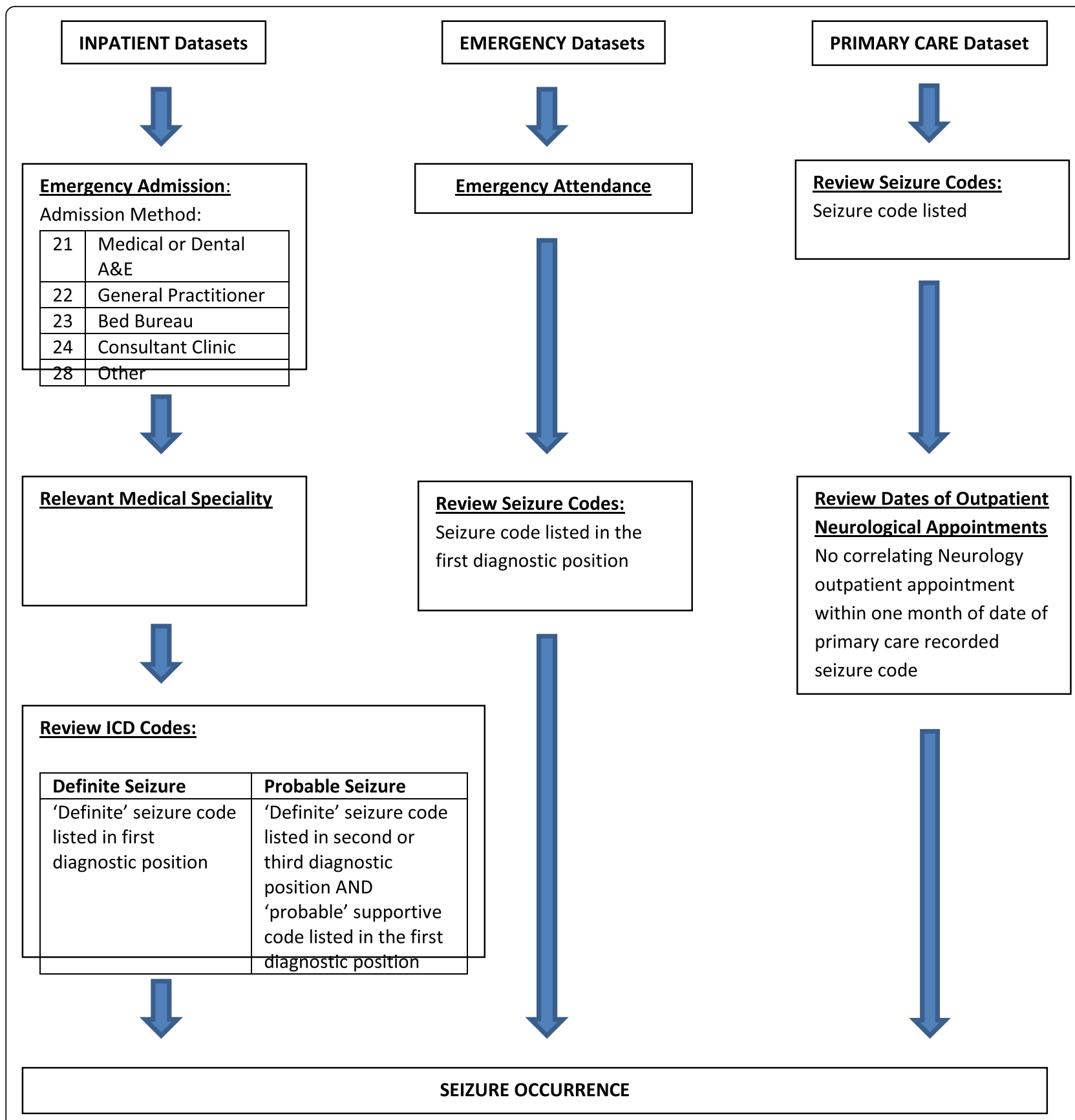

Fig. 1 Algorithm for the identification of seizure occurrence

sources provide more complete data for identifying first seizure occurrence, whilst primary care sources provide more complete data regarding the diagnosis of epilepsy and, in addition, prescribing information. For the analyses presented in this paper, the 'best case' dataset has been used including available data from all sources.

The results for each variable and outcome measure assessed are summarised in Table 2. Flowcharts summarising the identification of relevant data, Bland-Altman plots and Kaplan-Meier survival curves are presented in Supplementary Figures 6-34 and Supplementary Tables 7-14.

\section{Variables relevant to the identification of eligible} individuals and SANAD II baseline assessment

Sixty-two of the 98 included participants had a date (day, month and year) of first-ever seizure occurrence recorded in SANAD II during the time period covered by the available routine data and were eligible for the 
Table 1 Summary of primary and secondary care data*

\begin{tabular}{|c|c|c|c|c|c|}
\hline Variable & $\begin{array}{l}\text { Total } \\
\text { participants }\end{array}$ & $\begin{array}{l}\text { Total data in secondary } \\
\text { care datasets }\end{array}$ & $\begin{array}{l}\text { Total exclusively in } \\
\text { secondary care datasets }\end{array}$ & $\begin{array}{l}\text { Total data in primary } \\
\text { care datasets }\end{array}$ & $\begin{array}{l}\text { Total exclusively in } \\
\text { primary care datasets }\end{array}$ \\
\hline First-ever seizure (all types) & $12(52 \%)$ & $10(43 \%)$ & $6(26 \%)$ & $6(26 \%)$ & $2(8 \%)$ \\
\hline $\begin{array}{l}\text { Diagnosis of epilepsy } \\
\text { (baseline) }\end{array}$ & $18(78 \%)$ & $5^{* *}(22 \%)$ & $5(22 \%)$ & $13^{* * *}(57 \%)$ & $13(57 \%)$ \\
\hline Date of first follow-up seizure & $7(30 \%)$ & $3(13 \%)$ & $3(13 \%)$ & $4(17 \%)$ & $4(17 \%)$ \\
\hline Date of AED first prescription & $23(100 \%)$ & $0^{* * * *}$ & 0 & $23(100 \%)$ & $23(100 \%)$ \\
\hline
\end{tabular}

*For 23 participants in whom data from both primary and secondary care sources were available

**Diagnosis made by record of two seizure episodes

***Diagnosis made by record of a code consistent with 'diagnosis of epilepsy'

****Prescribing data only available in primary care datasets

assessment of 'date of first-ever seizure'. In the routine datasets, a first-ever seizure occurrence was identified in 23 of the 62 participants. The most common recorded codes were non-specific 'seizure' and 'epilepsy' codes. The most common ICD 10 code was 'Unspecified Convulsions (R568)', emergency code 'CNS Conditions - Epilepsy (HES 41, SAIL 17A)' and READ codes 'Convulsion NOS (R003z)' and 'Had a Fit (IB63)'.

Figure 2 shows a flowchart for the identification of 'first-ever seizures' in routine datasets. Although sixteen participants had a relevant attendance within $48 \mathrm{~h}$ of a seizure occurrence recorded in SANAD II, seizure could not be identified as the cause of the attendance due to inadequate or discrepant diagnostic codes. Codes included 'CNS, Non-Epilepsy' in the emergency datasets and 'Disorientation' and 'Confusion' in the inpatient datasets. The Bland-Altman plot (Fig. 3) demonstrates that when a date of first seizure is identified in the routinely collected data; agreement with data collected using CRFs is poor. The 95\% confidence limits of agreement between the dates of first-ever seizure are 145 and 313 days, well in excess of the specified 30 day clinically acceptable limit. The mean of the difference between the dates is 84 , indicating that on average the date of firstever seizure is identified in the routinely collected data 84 days after the seizure is identified in the SANAD II dataset. Limiting the first-ever seizures to 'tonic-clonic' seizures, the data were marginally more complete with seizures identified for 22 out of 43 participants with a tonic-clonic seizure recorded in the SANAD II dataset, although agreement regarding the date of first tonicclonic seizure occurrence remains poor.

At the time of recruitment into SANAD II, using routinely collected data 41 of 78 participants met the criteria for diagnosis of epilepsy and agreement was poor for the 'date of diagnosis of epilepsy'. Seizures could be classified in all participants using data retrieved from routinely recorded datasets; however, agreement was poor (Cohen's kappa 0.151, $P=0.018$ ) explained by the disproportionately large number of participants deemed 'unclassified' as a result of lack of clinical detail in the codes recorded.

\section{Variables and outcomes relevant to the follow-up in SANAD II}

Twenty-two participants had first follow-up seizures identified using routinely collected datasets, compared to 61 participants using SANAD II data. The mean time to first follow-up seizure was 325 days calculated using SANAD II data and 778 days calculated using routine data. Figure 4 presents the Kaplan-Meier curve. Proportionally, a greater number of first follow-up tonic-clonic seizures were identified, 20 participants using routinely collected data compared to 35 using SANAD II.

Data regarding adverse events were sparse in routine datasets, and of 97 adverse events recorded in SANAD II only two were identifiable in routine datasets.

Prescribing data were only available from the primary care dataset. Twenty-six 'first AED prescriptions' were identified, all prescription times being within the 90-day clinical limit of agreement with data recorded in SANAD II.

Dates of episodes of outpatient planned and inpatient and emergency unplanned healthcare resource use had fewer missing data compared to clinical variables; for example, 317 outpatient attendances were included in the SANAD II dataset, compared to 350 recorded in routinely collected data. Furthermore, the dates of attendance were within the acceptable clinical limits of agreement.

For some variables, additional data were recorded in the routinely recorded datasets that were not available in the SANAD II CRFs. For three participants in whom the CRF data indicated they were seizure-free, additional seizures were identified in the routine datasets. For one individual without an EEG result on the SANAD II CRF, the routine data indicated that they had an EEG. Two participants of the 23 with available prescribing data in routine datasets were prescribed additional AEDs not recorded in the CRFs. Finally, data regarding AED adherence could be inferred from the routine datasets using the frequency of repeat prescription, noting the assumptions made in reaching this result.

\section{Conclusions}

Routinely recorded data are increasingly being used in clinical trials to provide answers to important clinical 
Table 2 Summary of results: quality and agreement

\begin{tabular}{|c|c|c|c|c|c|c|}
\hline Variable & Total eligible & SANAD II total & Routine total & Difference $^{*}$ & Agreement** & $\begin{array}{l}\text { Acceptable } \\
\text { agreement }\end{array}$ \\
\hline \multicolumn{7}{|c|}{$\begin{array}{l}\text { Data variables relevant to the identification of eligible } \\
\text { individuals and baseline assessment in SANAD II }\end{array}$} \\
\hline First-ever seizure (all Types) & 62 & $62(100 \%)$ & $23(37.1 \%)$ & $P=0.002$ & $\begin{array}{l}\text { BA: }-84.09 \\
(-313.12-144.94)\end{array}$ & 30 days \\
\hline First-ever seizure (tonic-clonic) & 62 & $43(69.4 \%)$ & $22(35.5 \%)$ & $P=0.043$ & $\begin{array}{l}\text { BA: }-28.07 \\
(-123.72-67.58)\end{array}$ & 30 days \\
\hline Diagnosis of epilepsy (baseline) & 78 & $78(100 \%)$ & 37 (47.4\%) & $P=0.004$ & $\begin{array}{l}\text { BA: } 30.54 \\
(-106.17-167.25)\end{array}$ & 30 days \\
\hline Diagnosis of epilepsy (all-time) & 81 & $81(100 \%)$ & 47 (58.0\%) & $P=0.195$ & $\begin{array}{l}\text { BA: }-26.23 \\
(-294.10-241.64)\end{array}$ & 30 days \\
\hline Classification of seizures (baseline) & 37 & $37(100 \%)$ & 37 (100\%) & N/A & $\begin{array}{l}\text { CK: } 0.151 \\
(P=0.018)\end{array}$ & N/A \\
\hline Classification of seizures (all-time) & 47 & $47(100 \%)$ & $47(100 \%)$ & N/A & $\begin{array}{l}\text { CK: } 0.123 \\
(P=0.019)\end{array}$ & N/A \\
\hline $\begin{array}{l}\text { Clinical investigations } \\
\text { Magnetic resonance imaging }\end{array}$ & 98 & $72(73.5 \%)$ & $9(9.2 \%)$ & N/A & $\begin{array}{l}\text { CK: } 0.016 \\
(P=0.602)\end{array}$ & N/A \\
\hline $\begin{array}{l}\text { Clinical investigations } \\
\text { Computed tomography }\end{array}$ & 98 & $33(33.7 \%)$ & $27(27.6 \%)$ & N/A & $\begin{array}{l}C K: 0.406 \\
(P<0.001)\end{array}$ & N/A \\
\hline $\begin{array}{l}\text { Clinical investigations } \\
\text { Electroencephalography }\end{array}$ & 23 & $18(78.2 \%)$ & $8(34.8 \%)$ & N/A & $\begin{array}{l}\text { CK: } 0.188 \\
(P=0.131)\end{array}$ & N/A \\
\hline \multicolumn{7}{|c|}{ Data variables relevant to the follow-up in SANAD II } \\
\hline Date of first follow-up seizure & 98 & $61(62.2 \%)$ & $22(22.4 \%)$ & $P=0.024$ & $\begin{array}{l}\text { BA: }-86.26 \\
(-386.41-213.89)\end{array}$ & 30 Days \\
\hline Date of first follow-up tonic-clonic seizure & 98 & $35(35.7 \%)$ & $20(20.4 \%)$ & $P=0.374$ & $\begin{array}{l}\text { BA: }-9.20 \\
(-436.46-418.06)\end{array}$ & 30 Days \\
\hline Date 12-month remission achieved & 98 & $46(46.9 \%)$ & $74(75.5 \%)$ & $P=0.004$ & $\begin{array}{l}\text { BA: } 34.24 \\
(-115.48-183.96)\end{array}$ & 30 Days \\
\hline Date of AED first prescription & 26 & 26 & 25 & $P<0.001$ & $\begin{array}{l}\text { BA: }-19.76 \\
(-67.86-28.34)\end{array}$ & 90 Days \\
\hline Adverse events & 97 & 97 & 2 & - & - & - \\
\hline $\begin{array}{l}\text { Planned healthcare attendances } \\
\text { Baseline assessment }\end{array}$ & 98 & $98(100 \%)$ & $87(88.8 \%)$ & $P<0.001$ & $\begin{array}{l}\text { BA: } 1.67 \\
(-7.21-10.55)\end{array}$ & N/A \\
\hline $\begin{array}{l}\text { Planned healthcare attendances } \\
\text { Follow-up assessments }\end{array}$ & 350 & $350(100 \%)$ & $317(90.6 \%)$ & $P<0.14$ & $\begin{array}{l}\text { BA: } 0.07 \\
(-4.33-4.47)\end{array}$ & N/A \\
\hline Unplanned attendances: emergency & 94 & $52(55.3 \%)$ & 37 (39.4\%) & $P=0.051$ & $\begin{array}{l}\text { BA: } 0.05 \\
(-0.734-0.834)\end{array}$ & N/A \\
\hline Unplanned attendances: inpatient & 94 & $12(12.8 \%)$ & $19(20.2 \%)$ & $P=0.098$ & $\begin{array}{l}\text { BA: }-0.02 \\
(-0.72-0.68)\end{array}$ & N/A \\
\hline \multicolumn{7}{|c|}{ Outcomes relevant to the follow-up in SANAD II } \\
\hline Variable & Total eligible & SANAD II total & Routine total & $\begin{array}{l}\text { SANAD II mean } \\
(95 \% \mathrm{Cl})\end{array}$ & $\begin{array}{l}\text { Routine mean } \\
(95 \% \mathrm{Cl})\end{array}$ & Difference ${ }^{* * * *}$ \\
\hline Days to first follow-up seizure & 98 & $61(62.2 \%)$ & $22(22.4 \%)$ & $325(258-393)$ & $751(680-822)$ & $P<0.001$ \\
\hline Days to 12 -month remission & 98 & $46(46.9 \%)$ & $74(75.5 \%)$ & $567(515-618)$ & $393(375-410)$ & $P<0.001$ \\
\hline
\end{tabular}

*Paired $T$ test (normally distributed data), Wilcoxon signed-rank (non-normally distributed data)

**Bland-Altman methods (BA) (continuous data) = mean (lower 95\% confidence limit of agreement-upper 95\% confidence limit of agreement)

**Cohen kappa (CK) (categorical data) $=0.01-0.20$ as none to slight agreement, $0.21-0.40$ as fair, $0.41-0.60$ as moderate, $0.61-0.80$ as substantial and $0.81-1.00$ as almost perfect agreement

***Acceptable clinical limit of agreement specified a priori

****Log-rank test

questions. However, this study shows that for epilepsy, and potentially therefore for other chronic conditions, it is not currently possible to identify important clinical events and outcomes in routinely recorded data in the UK. Therefore, their exclusive use is not a valid substitute for data collected using standard methods such as case report forms completed at clinic visits or via telephone. There is an ongoing drive to incorporate routinely recorded data into RCTs in an effort to improve research efficiency and reduce the burden for 

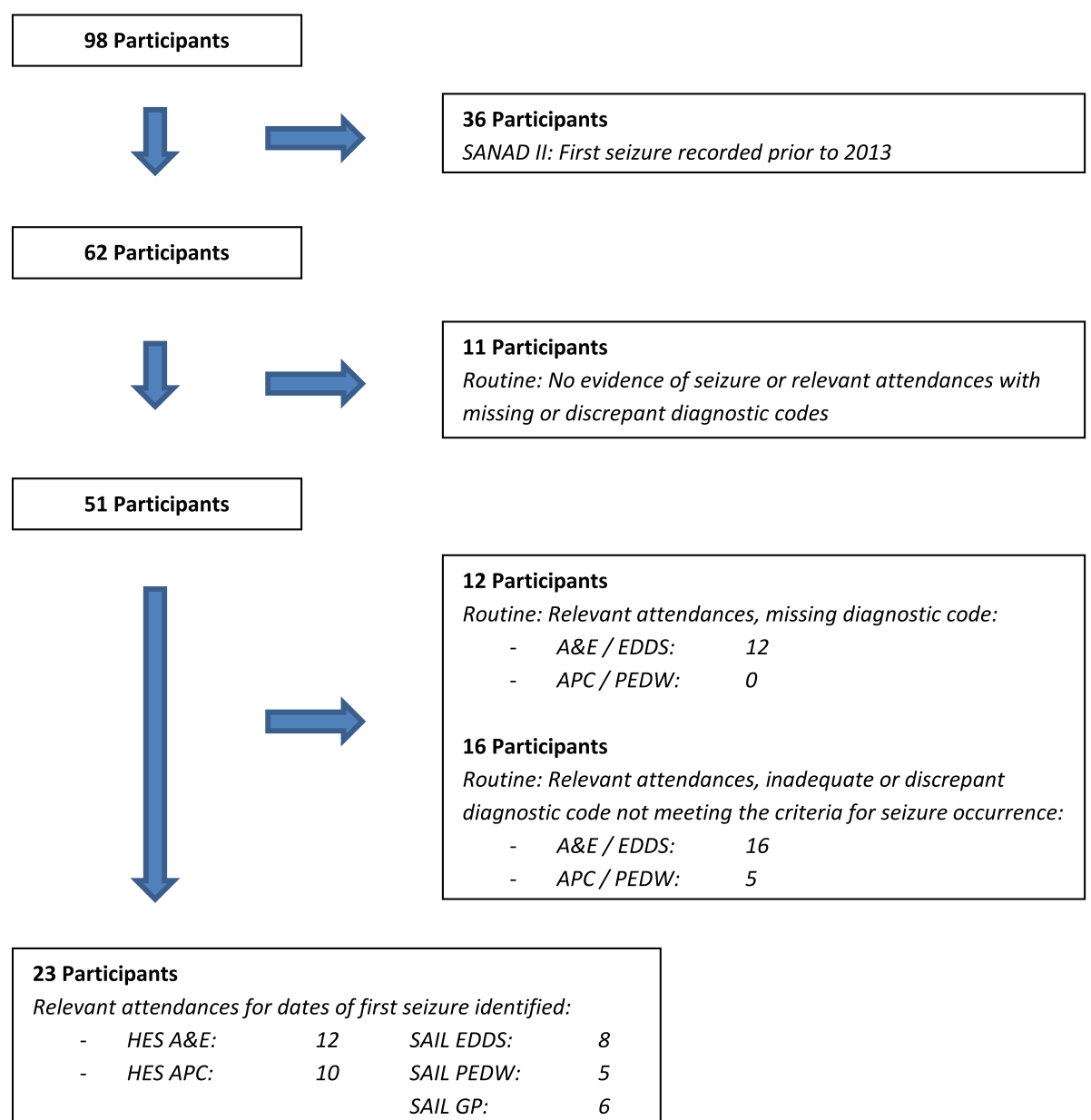

Fig. 2 The identification of the date of first-ever seizure in routine datasets

participants [1, 2], and the results of this study, using epilepsy as the exemplar, raise potentially significant concerns about the suitability of routine data for this purpose.

We assessed the use of routinely recorded data to identify individuals eligible for recruitment into a RCT for people with newly diagnosed epilepsy and to collect baseline, follow-up and outcome data.

Regarding seizure occurrence, it was not possible to identify baseline (pre-epilepsy diagnosis) seizures in the routinely collected data for $63 \%$ of patients or seizures during follow-up for $64 \%$. When baseline seizures or follow-up seizures were identified, there was a poor agreement with dates recorded in the trial database. The date of the first follow-up seizure identified was 86 days (mean) after the first follow-up seizure recorded in the trial database. Follow-up seizures could not be identified in the routine data, either because no event was documented at all, or because codes used did not indicate that seizure was the reason for attendance or admission.
As a consequence, analysis of routinely recorded data grossly underestimates the outcomes 'time to first seizure' and 'time to 12-month remission'.

Similarly, a 'diagnosis of epilepsy' was identified in less than half of the participants using the routinely recorded data around the time of randomisation into SANAD II. It was also not possible to classify seizure types for the majority of participants due to inadequate coding and coding options.

Specific codes labelling 'adverse events' were not recorded. Furthermore, healthcare attendances correlating with the dates of adverse events recorded in SANAD II were not identified. SANAD II participants self-reported more unplanned emergency attendances and fewer unplanned inpatient admissions, compared to those events identified in routinely recorded datasets. For these data, it may be more likely that the routinely recorded data are correct, and that recall bias is responsible for the discrepancy in the SANAD II dataset. 


\begin{tabular}{|l|l|}
\hline Mean & -84.09 \\
\hline Upper 95\% Confidence Limit of Agreement & 144.94 \\
\hline Lower 95\% Confidence Limit of Agreement & -313.12 \\
\hline
\end{tabular}

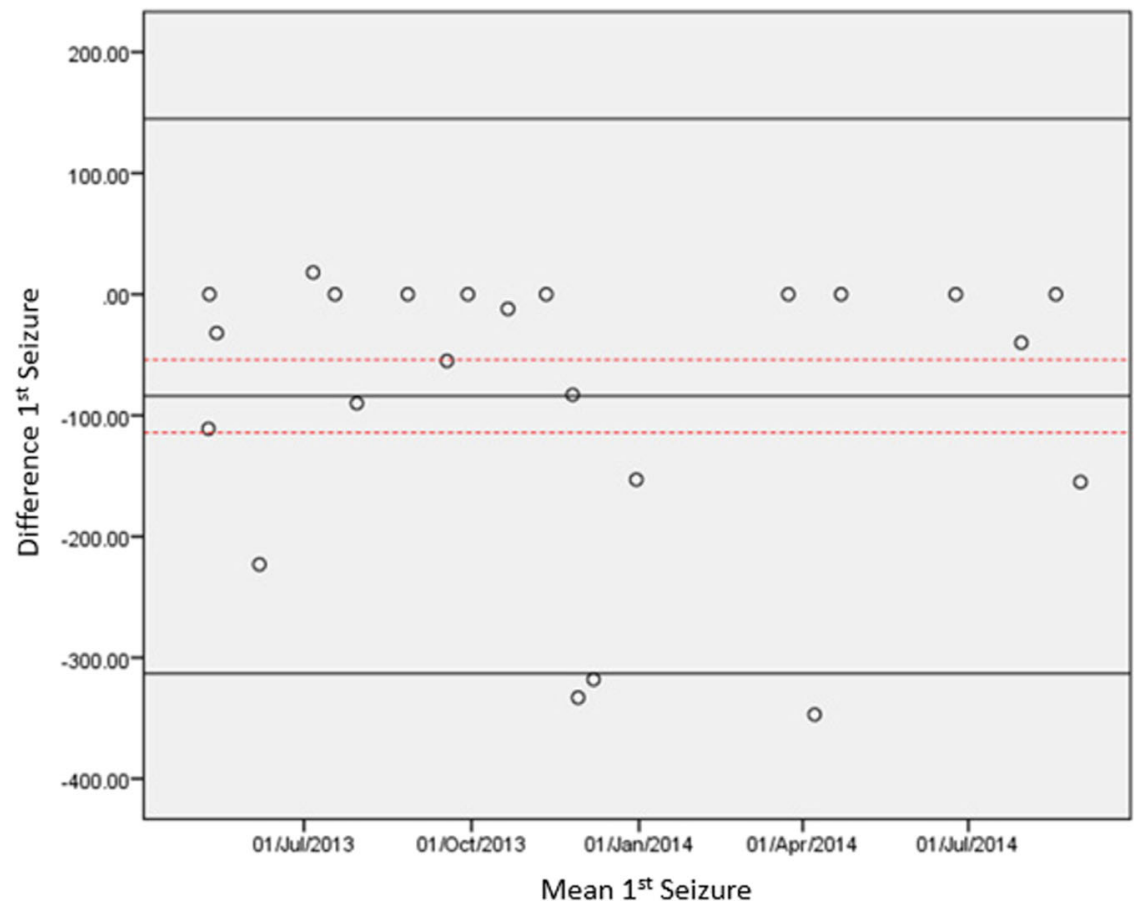

Fig. 3 Date of first-ever seizure: Bland-Altman plot

Explanations for the results may include the inaccurate recording of codes in routinely recorded datasets or inaccurate initial clinical diagnosis of seizures and epilepsy. Furthermore, certain events may not have been 'recordable', for example, if participants did not seek medical attention following seizure occurrence or if relevant codes or detail are not included in the available coding systems and routine datasets. Finally, patients with seizures may frequently be treated and then discharged from the emergency department, where diagnostic coding is not mandatory. This study report refers specifically to outcomes as applied to epilepsy, although it must be noted that in other disease areas, similar results may not be found and these explanations may not apply. For example, disease areas where the diagnosis is more explicit, where the presentation is acute and where admission (rather than discharge from an emergency department) is more commonly required may be more likely to be more completely and accurately recorded in routine datasets. Examples may include 'myocardial infarction' or 'stroke'.

The results of this study have implications for the use of routinely recorded data in RCTs in patients with epilepsy. In SANAD II, exclusively using routinely recorded data would not have allowed reliable identification of eligible individuals for recruitment, collection of baseline data or the collection of data for seizure and adverse outcomes [14, 30]. Furthermore, only 98 of 470 patients $(21 \%)$ provided consent for their routine data to be retrieved. Notably, the 470 patients had already consented to participate in the SANAD II RCT.

Whilst routinely recorded data could not be used alone to construct clinical outcomes, routine data could aid the identification of events, such as seizure occurrence or prescription changes, that had been missed, and these data remain important for assessing health service resource use for economic analyses [31]. However, the limitations in accessing these data, such as cost and lengthy application processes, would need to be considered [17].

Further research is urgently required to assess the attributes, additional benefits, feasibility and costeffectiveness of accessing routinely recorded data during RCTs in the UK in epilepsy and other common disease areas. The 'Studies Within A Trial' (SWAT) approach, embedding methodological research within an existing 


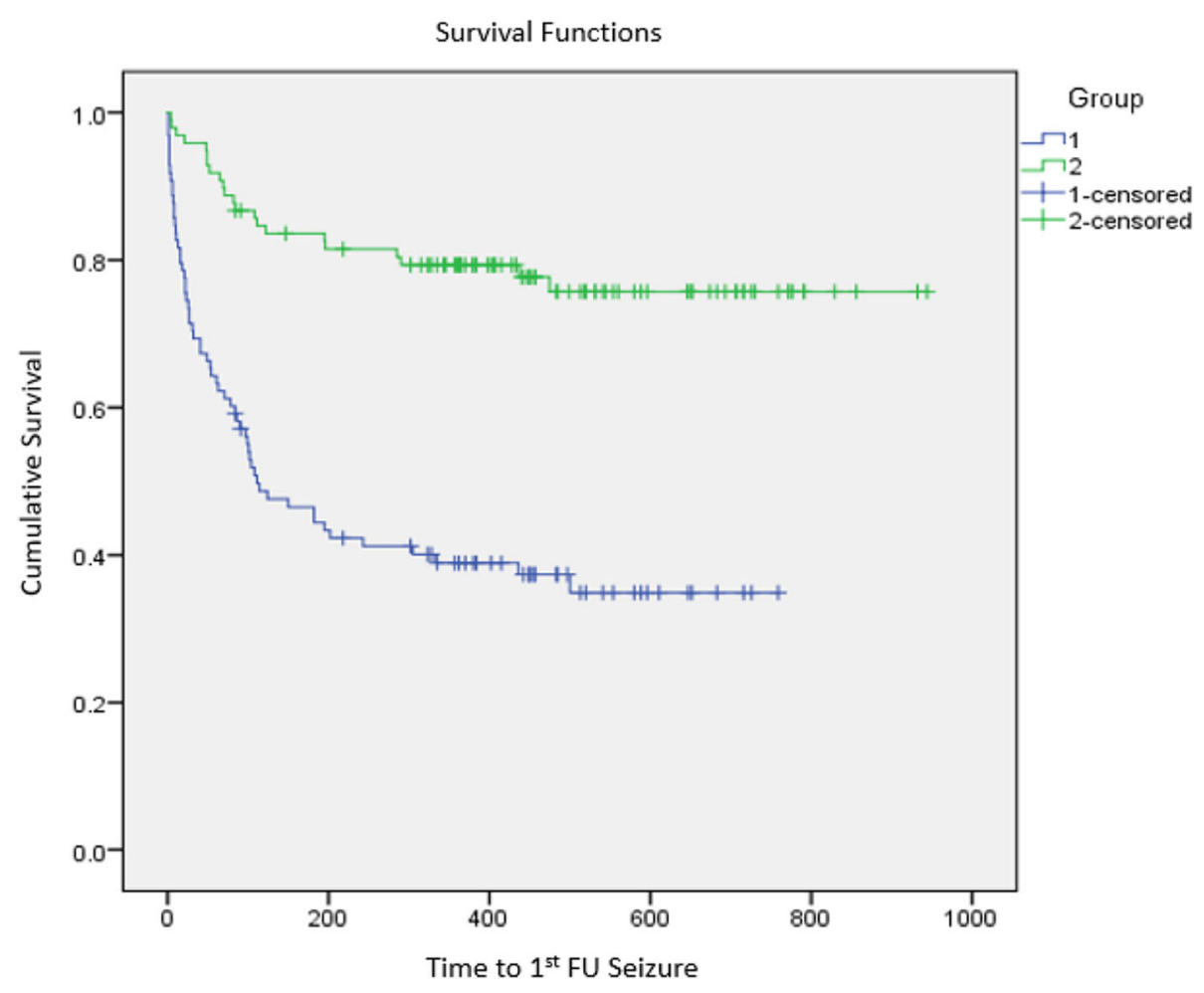

Fig. 4 Kaplan-Meier curve: the time to first follow-up seizure

prospective trial [32], could be suggested as the method to facilitate such research. For example, routinely recorded data could be requested for a subset of participants within existing RCTs. The analysis of such data, compared to the standard RCT methods, may directly inform the use of routine data in the ongoing RCT as well as inform future research.

The 'de-identified' nature of primary care data, cost and poor geographical coverage were notable limitations. Development of the infrastructure to record national primary care data coverage is required, either through improved collaboration between existing routine data sources with individual-level data linkage or the development of national data sources, such as the NHS Digital General Practice Extraction Service [33]. Further, a number of countries have integrated healthcare systems allowing for national administrative healthcare databases, such as the Swedish Hospital Discharge Register, the Danish National Hospital Register and the Canadian Chronic Disease Surveillance System. In these examples, it is possible to retrieve routinely recorded data from electronic medical records for individuals across hospital inpatient admissions and emergency care, outpatient clinic and primary care attendances. In the UK, standardisation of the coding systems used in different care settings and between datasets and greater involvement of clinicians in the clinical coding process may improve the accuracy and completeness of clinical coding. A suggested proposed improvement could include the development of a national, integrated electronic health record for use in direct clinical care, with secondary uses in audit and research. Such a proposal would include direct clinician input and selection of diagnostic codes and would have significant potential for the improved recording of data and improved utility for the datasets in clinical practice and research.

Whilst patients and the public are broadly supportive of data recording and sharing for healthcare research, concerns remain over confidentiality and potential abuses of data [34]. Public concerns regarding the sharing and linking of routinely recorded data will hamper future efforts to develop linked routinely recorded administrative databases, despite the likely benefits to individuals and the population. Further research is required with public engagement to define the issues of importance to members of the public and assess perspectives with regards to the routine recording of data and subsequent use for secondary purposes including research.

This study has notable limitations. The variables and constructed outcomes derived from the routinely recorded datasets were defined and extracted using algorithms developed for each comparison. There is a 
risk that relevant clinical events may not have been identified. To address this limitation and explore the data further, the routinely recorded data for each participant were examined in their entirety to identify additional relevant events. This process was feasible as a result of the small sample size. Furthermore, this study involved the retrospective identification of events in routinely recorded datasets and comparison to events identified in a study designed and completed using standard prospective methods. It must be acknowledged that the comparator study was not designed to be completed retrospectively using routinely recorded datasets, and if this had been the case, alternative methodologies may have been employed.

Substantial further development is now required to improve the utility of routinely recorded data in research. To improve the likelihood of significant progress, initiatives for development should include collaboration from the government, National Health Service, researchers and perhaps most importantly acknowledging recent controversies, patients and the public; re-gaining their trust will be essential in realising the individual and population healthcare benefits of routinely recorded data. Examples of such work in progress include the European Health Data and Evidence Network [35], whose objectives include standardising real-world health data Europe-wide for purposes including research, Health Data Research UK [36] and the PED4PED initiative, a project aiming to improve outcomes for patients with epilepsy by linking data between primary, secondary and the emergency services [37].

\section{Abbreviations \\ AED: Antiepileptic drug; CNS: Central nervous system; CRF: Case report form; HES: Hospital Episode Statistics; NHS: National Health Service; RCT: Randomised controlled trial; SAIL: Secure Anonymised Information Linkage Databank; SANA D II: The Standard and New Antiepileptic Drug Trial II}

\section{Supplementary Information}

The online version contains supplementary material available at https://doi. org/10.1186/s13063-021-05294-6.

Additional file 1.

Additional file 2.

Acknowledgements

Not applicable.

\section{Transparency statement}

The lead author (GA Powell) affirms that the manuscript is an honest, accurate and transparent account of the study being reported; that no important aspects of the study have been omitted; and that any discrepancies from the study as planned (and, if relevant, registered) have been explained.

\section{Authors' contributions}

GAP performed the background research, prepared the protocol, liaised with the routine data sources, prepared and submitted the routine data applications, analysed the data and drafted and redrafted the manuscript.
LB, CTS, DAH, PRW and AGM provided input into the development of the protocol and analysis plan and drafted and redrafted the manuscript. AGM is the guarantor for the report. The University of Liverpool was the sponsor for the study. The corresponding author attests that all listed authors meet authorship criteria and that no others meeting the criteria have been omitted. The authors read and approved the final manuscript.

\section{Funding}

This report is independent research arising from a Clinical Training Fellowship (GA Powell) awarded by the Medical Research Council Hubs for Trials Methodology Research (MRC HTMR) (MR/L004933/2/P16). PW is funded by the MRC HTMR (North West Hub Grant Code: MR/K025635/1). The views expressed are those of the authors and not necessarily those of the NHS, the Medical Research Council or the data sources detailed in the report. The funding body had no role in the design of the study, collection, analysis and interpretation of data or in writing the manuscript.

\section{Availability of data and materials}

The study dataset (consisting of SANAD || RCT data and routinely recorded data provided by NHS Digital and SAIL) cannot be shared. This is a result of the risks to individual confidentiality and data security arrangements detailed in the relevant Data Sharing Agreements.

\section{Declarations}

Ethics approval and consent to participate

To request identifiable data from routine data sources, participant consent was required. Participant Information Leaflets were provided and informed written consent obtained from all study participants. The North of Scotland Research Ethics Service (16/NS/0007) and Health Research Authority (IRAS 189002) approved the study.

\section{Consent for publication}

Not applicable.

\section{Competing interests}

All authors have completed the ICMJE uniform disclosure form at wwW. icmje.org/coi_disclosure.pdf and declare: This report is independent research arising from a Clinical Training Fellowship (GA Powell) awarded by the Medical Research Council Hubs for Trials Methodology Research (MRC HTMR) (MR/L004933/2/P16). PW is funded by the MRC HTMR (North West Hub Grant Code: MR/K025635/1). GP, LB, CTS, DAH, PRW and AGM have no financial relationships with any organisations that might have an interest in the submitted work in the previous 3 years and no other relationships or activities that could appear to have influenced the submitted work.

\section{Author details}

${ }^{1}$ Department of Molecular and Clinical Pharmacology, Clinical Sciences Centre, Lower Lane, Fazakerley, Liverpool L9 7LJ, UK. ${ }^{2}$ Department of Biostatistics, University of Liverpool, Waterhouse Building, Block F, 1-5 Brownlow Street, Liverpool L69 3GL, UK. ${ }^{3}$ Liverpool Health Partners, Liverpool L69 3GL, UK. ${ }^{4}$ Centre for Health Economics \& Medicines Evaluation, Bangor University, Ardudwy, Normal Site, Gwynedd, North Wales LL57 2PZ, UK.

Received: 16 August 2020 Accepted: 24 April 2021

Published online: 05 July 2021

\section{References}

1. Medical Research Council. MRC Digital Innovation Hubs. 2019 11/10/2019; Available from: https://mrc.ukri.org/news/browse/mrc-to-run-competitionprocess-for-digital-innovation-hubs/.

2. UK Department of Health. New heart disease drug to be made available for NHS patients 2020 [cited 2020 9/3/20]; Available from: https://www.gov.uk/government/news/new-heart-disease-drug-to-be-ma de-available-for-nhs-patients.

3. McKee M. Routine data: a resource for clinical audit? Qual Health Care. 1993; 2(2):104-11. https://doi.org/10.1136/qshc.2.2.104.

4. NHS England. NHS Payment System. 2016 [cited 2016 18/07/2016]; Available from: (http://www.england.nhs.uk/resources/pay-syst).

5. Garrett E, B.H., Dibbon C Health Administrative Data: exploring the potential for academic research. 2010. 
6. Friedman CP, et al. Toward a science of learning systems: a research agenda for the high-functioning Learning Health System. J Am Med Inform Assoc. 2015;22(1):43-50. https://doi.org/10.1136/amiajnl-2014-002977.

7. Buchbinder R, Bombardier C, Yeung M, Tugwell P. Which outcome measures should be used in rheumatoid arthritis clinical trials? Arthritis Rheum. 1995;38(11):1568-80. https://doi.org/10.1002/art.1780381108.

8. COMET Initiative. Core Outcome Measures in Effectiveness Trials. 2020 05/ 03/2020; Available from: http://www.comet-initiative.org/.

9. McGregor J, et al. The Health Informatics Trial Enhancement Project (HITE): Using routinely collected primary care data to identify potential participants for a depression trial. Trials [Electronic Resource]. 2010;11:39.

10. Lewsey JD, et al. Using routine data to complement and enhance the results of randomised controlled trials. Health Technol Assess. 2000;4(22):i+ iii-iv+1-45.

11. Williams JG, et al. Can randomised trials rely on existing electronic data? A feasibility study to explore the value of routine data in health technology assessment. Health Technol Assess (Winchester, England). 2003;7(26):iii $v-x, 1-117$.

12. Gulliford MC, van Staa TP, McDermott $L$, et al. Cluster randomized trials utilizing primary care electronic health records: methodological issues in design, conduct, and analysis (eCRT Study). Trials. 2014;15:220. https://doi. org/10.1186/1745-6215-15-220.

13. Franklin $\mathrm{M}$, Thorn J. Self-reported and routinely collected electronic healthcare resource-use data for trial-based economic evaluations: the current state of play in England and considerations for the future. BMC Med Res Methodol. 2019;19:8. https://doi.org/10.1186/s12874-018-0649-9.

14. Loke YK. Use of databases for clinical research. Arch Dis Child. 2014;99(6): 587-9. https://doi.org/10.1136/archdischild-2013-304466.

15. Healy P, Galvin S, Williamson PR, et al. Identifying trial recruitment uncertainties using a James Lind Alliance Priority Setting Partnership - the PRioRiTy (Prioritising Recruitment in Randomised Trials) study. Trials. 2018;19: 147. https://doi.org/10.1186/s13063-018-2544-4

16. Raftery J, Roderick P, Stevens A. Potential use of routine databases in health technology assessment. Health Technol Assess (Winchester, England). 2005; 9(20):1-92 iii-iv.

17. Powell GA, Bonnett $L J$, Tudur-Smith C, et al. Using routinely recorded data in the UK to assess outcomes in a randomised controlled trial: The Trials of Access. Trials. 2017;18:389. https://doi.org/10.1186/s13063-017-2135-9.

18. Tan M, Wilson I, Braganza V, Ignatiadis S, Boston R, Sundararajan V, et al. Development and validation of an epidemiologic case definition of epilepsy for use with routinely collected Australian health data. Epilepsy Behav. 2015; 51:65-72. https://doi.org/10.1016/j.yebeh.2015.06.031.

19. Kilburn LS, Aresu M, Banerji J, et al. Can routine data be used to support cancer clinical trials? A historical baseline on which to build: retrospective linkage of data from the TACT (CRUK 01/001) breast cancer trial and the National Cancer Data Repository. Trials. 2017;18:561. https://doi.org/10.1186/ s13063-017-2308-6.

20. NHS Digital. The Health and Social Care Information Centre. 2016 [cited 2016 16th July]; Available from: http://www.hscic.gov.uk.

21. SAIL. The Secure Anonymised Information Linkage Databank. 2016 [cited 2016 01/06/2016]; Available from: http://www.saildatabank.com.

22. World-Health-Organisation. International Statistical Classification of Diseases and Related Health Problems (ICD) 10. 2016 [cited 2016 18/ 07/2016]; Available from: http://apps.who.int/classifications/icd10/ browse/2016/en.

23. NHS Digital. UK READ Codes. 2017 [cited 2017 21/03/17]; Available from: https://data.gov.uk/dataset/uk-read-code.

24. Grainger $R$, Pearson $M$, Dixon $P$, et al. Referral patterns after a seizure admission in an English region: an opportunity for effective intervention? An observational study of routine hospital data. BMJ Open. 2016;6:e010100. https://doi.org/10.1136/bmjopen-2015-010100.

25. Walker PP, Thompson E, Crone H, Flatt G, Holton K, Hill SL, et al. Use of mortality within 30 days of a COPD hospitalisation as a measure of COPD care in UK hospitals. Thorax. 2013:68(10):968-70. https://doi.org/10.1136/ thoraxjnl-2012-202365.

26. Shawihdi M, Thompson E, Kapoor N, Powell G, Sturgess RP, Stern N, et al. Variation in gastroscopy rate in English general practice and outcome for oesophagogastric cancer: retrospective analysis of Hospital Episode Statistics. Gut. 2014;63(2):250-61. https://doi.org/10.1136/gutjnl-2012-304202.
27. Abraham KA, Thompson EB, Bodger K, Pearson M. Inequalities in outcomes of acute kidney injury in England. QJM. 2012;105(8):729-40. https://doi.org/1 0.1093/qjmed/hcs037.

28. Altman D. Practical Statistics for Medical Research: Chapman and Hall; 1990. p. 624

29. Bland JM, Altman DG. Measuring agreement in method comparison studies. Stat Methods Med Res. 1999;8(2):135-60. https://doi.org/10.1177/09622802 9900800204.

30. Fairweather NB, Rogerson S. A moral approach to electronic patient records. Med Informatics Internet Med. 2001;26(3):219-34. https://doi.org/10.1080/14 639230110076412

31. Appleyard SE, Gilbert DC. Innovative Solutions for Clinical Trial Follow-up: Adding Value from Nationally Held UK Data. Clin Oncol. 2017;29(12):789-95. https://doi.org/10.1016/j.clon.2017.10.003.

32. Medical Research Council Hubs for Trials Methodology Research. Studies Within A Trial. 2018 6/2/18; Available from: http://www.methodologyhubs. mrc.ac.uk/resources/swat.

33. NHS Digital. General Practice Extraction Service. 2016 [cited 2016 05/04/ 2016]; Available from: http://www.hscic.gov.uk/gpes.

34. Kalkman S, van Delden J, Banerjee A, Tyl B, Mostert M, van Thiel G. Patients' and public views and attitudes towards the sharing of health data for research: a narrative review of the empirical evidence. J Med Ethics. 2019. https://doi.org/10.1136/medethics-2019-105651.

35. European Health Data and Evidence Network. European Health Data and Evidence Network: Vision and Mission. 2021 [cited 2021 21/04/21]; Available from: https://wwwehden eu/vision-and-mission/.

36. Health Data Research UK. Health Data Research UK is uniting the UK's health data to enable discoveries that improve people's lives. 2020 12/03/ 20; Available from: https://www.hdruk.ac.uk/about/.

37. Health Data Research UK. PED4PED - People with Epilepsy sharing Data for care with Paramedics and the Emergency Department. 2020 12/03/20; Available from: https://www.hdruk.ac.uk/projects/ped4ped/.

\section{Publisher's Note}

Springer Nature remains neutral with regard to jurisdictional claims in published maps and institutional affiliations.

Ready to submit your research? Choose BMC and benefit from:

- fast, convenient online submission

- thorough peer review by experienced researchers in your field

- rapid publication on acceptance

- support for research data, including large and complex data types

- gold Open Access which fosters wider collaboration and increased citations

- maximum visibility for your research: over $100 \mathrm{M}$ website views per year

At $\mathrm{BMC}$, research is always in progress.

Learn more biomedcentral.com/submissions 